\title{
Study of skin and nail Trichosporon species as a normal flora based on age groups in healthy persons in Tehran-Iran
}

\begin{abstract}
The skin is the body's largest organ that hosts heterogeneous inhabitants. Until now, the diversity of the cutaneous microbiome was mainly investigated for bacteria and there is a little information about the skin fungal flora and Trichosporon is occasionally found as normal flora of skin. In this study, differences in Trichosporon community structure associated with 9 different skin sites of 238 healthy people during 10 months from July to March 2016, are described. These subjects were divided by age into 4 groups: infants, children, adults and geriatrics. The collected samples were examined by culture on Sabouraud Chloramphenicol Agar and Sabouraud Cycloheximide Chloramphenicol Agar. For precise identification of isolates in the species level, ITS1-5. 8S-ITS2 $r$ DNA regions were sequenced. The frequency of Trichosporon species was not significantly different between age groups. The most Trichosporon isolations were related to the adult age group and the fewest in the infants. T.asahii was the predominant isolated species in all age groups. This study showed statistically significant effect of the subjects' sex on Trichosporon population resident on human skin surface.
\end{abstract}

Volume 8 Issue 2 - 2019

\author{
Zahra Rafat,' Seyed Jamal Hashemi,' Roshank \\ Daie Ghazvini,' Mohammad Yarahmadi,' \\ Maryam Sharafkhah ${ }^{2}$ \\ 'Department of Medical Parasitology and Mycology, School of \\ Public Health, Tehran University of Medical Sciences, Iran \\ ${ }^{2}$ Department of Epidemiology and Biostatistics, School of Public \\ Health, Tehran University of Medical Sciences, Iran
}

\begin{abstract}
Correspondence: Seyed Jamal Hashemi, Department of Medical Parasitology and Mycology, School of Public Health, Tehran University of Medical Sciences, Tehran, Iran, Tel +98- 21
\end{abstract} 4293 3150, Fax +98 21 88951392, Email sjhashemi@tums.ac.ir

Received: September 20, 2018| Published: March 22, 2019

Keywords: skin residents, cutaneous Trichosporon composition, different age groups, DNA-sequencing, culture, Microbial epidemiology, Irant

\section{Introduction}

Skin as the largest organ of human body is significantly colonized by a variety of bacterial and fungal population ${ }^{1,2}$ and richosporon is a normal fungal inhabitant of the skin. Opportunistic infections by this fungus has been increasingly often report in immune compromised patients. $^{3-6}$ Two of the most factors influencing the complex of inhabitants on the skin are gender ${ }^{7}$ and age. ${ }^{8,9}$ There is little information about Trichosporon distribution inhabiting on human skin in the world and it should be noted, this is the first study about this subject in Iran. The purpose of this study were to create new information about the type and percentage of Trichosporon population in each site of the human skin, attempting to determine what influences the individual's age and sex have on the composition and percentage of cutaneous Trichosporon community in Iranian people. As we know, Iran is a tropical country and fungal organisms can significantly growth over this condition.

\section{Materials and Methods}

\section{Ethics statement}

This study was approved by ethical committee of Tehran University of medical science (the number of Ethics Committee protocol: IR.TUMS.SPH.REC.1395.1339). A written informed consent was obtained from all subjects or their guardians prior to sample collection. All data were de-identified.

\section{Sampling}

A total of 238 healthy people, in four age groups, were studied. These subjects were including 119 males and 119 females, with equal gender distribution in all age groups. All samples were selected from 5 areas of Tehran (North, South, East, West and Center of this city). The distribution of subjects in each group was as follows:

a) 55 healthy, full term babies between the ages of 4 to 15 days, including infants referring to the health house.

b) 60 children aged between 1 and 12 years old. Most were drawn from schools and kindergartens.

c) 62 healthy adults, aged between 18 and 45 years old. This group was composed of students of Tehran University, factory workers, health workers and house wives.

d) 61 old people over 60 years age which often consist of retirements.

At the time of sampling, the age and gender of each subject were recorded. The subjects haven't washed their hands, feet, or other areas just before sampling and the sampling was made after the activities of the day. The following areas were sampled by means of a cotton-tipped swab moisted with sterile serum physiology: the forehead, dorsum of hands, dorsum of feet, finger nails, toenails, the axilla, the groin, the interdigital spaces of hand and foot and the sub mammary space in women. All swabs were cultured on Sabouraud Chloramphenicol Agar (SC, Merck, Germany). All cultures were incubated in $25^{\circ} \mathrm{C}$ for 4 days. Isolated colonies then were cultured on Sabouraud Dextrose Agar with chloramphenicol and cycloheximide (Micro media, Hungary) in $25^{\circ} \mathrm{C}$ after 4 days. This medium is a selective solid medium for differentiation Trichosporon spp from Geothricum candidum. Unlike Geothricum candidum, Trichosporon isolates grow on this medium. In this study for a correct determination of isolates in the species level, DNA sequencing was performed.

\section{Molecular technique}

DNA Extraction: An aliquot of $100 \mu \mathrm{L}$ of cell suspension was 
transferred to micro tubes and incubated at $100^{\circ} \mathrm{C}$ in a boiling waterbath for $10 \mathrm{~min}$, then centrifuged at 5,000 $\mathrm{g}$ for 5 minutes. The upper aqueous layer (containing the DNA) was carefully transferred to a clean tube and was used for PCR.

PCR conditions and sequencing: PCR amplification of ITS1-5. 8S-ITS2 rDNA regions were performed. ${ }^{15}$ Positive PCR products were sent for sequencing at Bioneer Advanced Nucleic Acids core facility. The ITS sequences were then parsed from the coating and separately used to perform individual nucleotide-nucleotide searches using the BLASTn algorithm at the NCBI website (http://www/ncbi.nlm.nih. gov/BLAST/). Fungal identifications were made based on maximum identities $\geq 99 \%$ and query coverage $\geq 98 \%$ with this method.

Statistical tests: In this study, one tail chi-square test was performed for each analysis.

\section{Results}

In this study, on each medium that the growth of Trichosporon was positive, one isolate was identified and for precise identification of isolates in the species level, DNA sequencing was performed. Among the studied population, divided into 4 groups by age including infants, children, adults and the elderly, 12 persons were positive for Trichosporon isolation. The highest prevalence of Trichosporon isolation was related to the adults age group $(n=7,58.33 \%)$ and the lowest prevalence was related to the infants $(\mathrm{n}=1,8.33 \%)$ and showed the age of subjects was not significantly effective on cutaneous Trichosporon community $(\mathrm{p}>0.01)$. Also in this study

T. asahii $(\mathrm{n}=11,91.66 \%)$ among all species was predominant in all age groups follow by T.coremiiforme $(n=1,9.09)$. It should be noted there was not a significant difference between isolated species according the age (Table 1). In this study 238 healthy people including 119 males and 119 females were examined. From 12 persons who were positive for Trichosporon isolation, the isolation of Trichosporon was significantly different between males $(\mathrm{n}=4,33.33 \%)$ and females $(\mathrm{n}=8,66.66 \%)$ and there was significant difference in cutaneous Trichosporon population between the genders. $(\mathrm{p}=0.01)($ Table 2$)$.

Table I The frequency of Trichosporon species in different age groups

\begin{tabular}{|c|c|c|c|c|c|c|c|c|c|c|}
\hline Age group & Infants & & Children & & Adults & & Elderly & & Total & \\
\hline Trichosporon species & Number & $\%$ & Number & $\%$ & Number & $\%$ & Number & $\%$ & Number & $\%$ \\
\hline T. asahii & 0 & 0 & 1 & 100 & 7 & 100 & 3 & 100 & $\mathrm{II}$ & 91.66 \\
\hline T.coremiiforme & I & 100 & 0 & 0 & 0 & 0 & 0 & 0 & I & 8.33 \\
\hline Total & I & 100 & I & 100 & 7 & 100 & 3 & 100 & 12 & 100 \\
\hline
\end{tabular}

Table 2 The frequency of Trichosporon species in regard to gender

\begin{tabular}{lllllll}
\hline Gender & Men & & Women & \multicolumn{3}{c}{ Total } \\
\hline Trichosporon species & Number & $\%$ & Number & $\%$ & Number & $\%$ \\
T.asahii & 4 & 100 & 7 & 87.5 & 11 & 91.66 \\
T.coremiiforme & 0 & 0 & 1 & 12.5 & 1 & 8.33 \\
Total & 4 & 100 & 8 & 100 & 12 & 100 \\
\hline
\end{tabular}

Considering Trichosporon isolation in regard to anatomic sites, from 19 positive sites for Trichosporon isolation, the highest prevalence of Trichosporon isolation was related to the axilla $(n=4$, $21.5 \%)$ and the groin $(n=4,21.5 \%)$ and the lowest prevalence was related to the sub mammary space in women $(n=0,0 . \%)$ and dorsum of hands $(n=0,0 . \%)$. Also in this study, T.asahii $(n=15,78.94 \%)$ showed the highest prevalence of Trichosporon isolations in all studied anatomical sites (Table 3).

Table 3 The frequency of Trichosporon species in regard to anatomic site of the body

\begin{tabular}{|c|c|c|c|c|c|c|}
\hline \multirow{2}{*}{$\begin{array}{l}\text { Trichosporon species } \\
\text { Anatomic site of body }\end{array}$} & \multicolumn{2}{|l|}{ T.asahii } & \multicolumn{2}{|c|}{ T.coremiiforme } & \multicolumn{2}{|l|}{ Total } \\
\hline & Number & $\%$ & Number & $\%$ & Number & $\%$ \\
\hline Forehead & I & 6.66 & 1 & 25 & 2 & 10.52 \\
\hline Dorsum of hands & 0 & 0 & 0 & 0 & 0 & 0 \\
\hline Dorsum of feet & I & 6.66 & 0 & 0 & I & 5.26 \\
\hline Finger nail & 3 & 20 & 0 & 0 & 3 & 15.78 \\
\hline Toe nail & 3 & 20 & 0 & 0 & 3 & 15.78 \\
\hline Axilla & 3 & 20 & 1 & 25 & 4 & 21.05 \\
\hline Groin & 3 & 20 & 1 & 25 & 4 & 21.05 \\
\hline Inter digital space of feet & I & 6.66 & 1 & 25 & 2 & 10.52 \\
\hline Sub mammary space & 0 & 0 & 0 & 0 & 0 & 0 \\
\hline total & 15 & 100 & 4 & 100 & 19 & 100 \\
\hline
\end{tabular}




\section{Discussion}

In this study, we characterized the cutaneous Trichosporon composition and the percentage of Trichosporon species inhabiting on each site of human skin by focus on show the effect of the individual's age and sex on it. Our study showed that the lowest prevalence of Trichosporon isolation is related to the infant age group, this finding may be a result of this fact that the cases were at the age of 4-15 days and the chance of skin colonization with Trichosporon during this time is less than other age groups. Fetal skin is sterile, but Trichosporon colonization occurs through the hands of health care workers, parents and the infants contact with objects and equipment related to neonatal care. ${ }^{10,11}$ The highest prevalence of Trichosporon isolation recorded from the skin of adult individuals. The main reason for this is possibly due to high level of daily activities in this age group and their greater contact with source of pollution compared with another age groups. ${ }^{12}$ In our study the frequency of Trichosporon isolation was significantly greater in females compared to males. Physiological and anatomical differences between male and female and cutaneous environments such as sweat, sebum and hormone production, partially account for the microbial differences seen between the genders. ${ }^{13}$ During this investigation the maximum amount of Trichosporon isolation in regard to anatomic site of the skin was related to the axilla and the groin. These regions are higher in temperature and humidity, which encourages the growth of these yeasts, the increased chance of yeasts isolation from this anatomical site is explainable. ${ }^{14}$ Isolation of T.asahii as the commonest species in all sites of the skin in our study was noteworthy. The prognosis of infection caused by this yeast is very poor, its mortality being approximately $70 \%$. This is higher than that of candidacies, with a mortality of $40 \%$.

\section{Acknowledgments}

The authors appreciate the support of Tehran University of Medical Sciences, Tehran, Iran.

\section{Conflicts of interest}

Authors declare that there is no conflict of interest.

\section{References}

1. Grice EA, Segre JA. The skin microbiome. Nature Reviews Microbiology. 2011;9(4):244-253.
2. Takemoto A, Cho O, Morohoshi Y, et al. Molecular characterization of the skin fungal microbiome in patients with psoriasis. The Journal of dermatology. 2015;42(2):166-1670.

3. Takeuchi T, Kobayashi M, Moriki T, et al. Application of a monoclonal antibody for the detection of Trichosporon beigelii in paraffin-embedded tissue sections. The Journal of pathology. 1988;156(1):23-27.

4. Mochizuki T, Sugiura H, Watanabe S, et al. A case of disseminated trichosporonosis: a case report and immunohistochemical identification of fungal elements. Journal of Medical and Veterinary Mycology. 1988;26(6):343-349.

5. Walling DM, McGraw DJ, Men WG, et al. Disseminated infection with Trichosporon beigelii. Reviews of infectious diseases. 1987;9(5):1013109.

6. Alegre A, Algora M, Penalver M, et al. Focal hepato-splenic mycosis caused by Trichosporon beigelii in a patient with acute leukemia. Sangre. 1991;36(4):311-417.

7. Fierer N, Hamady M, Lauber CL, et al. The influence of sex, handedness, and washing on the diversity of hand surface bacteria. Proceedings of the National Academy of Sciences. 2008;105(46):17994-17999.

8. Blaser MJ, Dominguez-Bello MG, Contreras M, et al. Distinct cutaneous bacterial assemblages in a sampling of South American Amerindians and US residents. The ISME journal. 2013;7(1):85-95.

9. Ying S, Zeng D-N, Chi L, et al. The influence of age and gender on skinassociated microbial communities in urban and rural human populations. PloS one. 2015;10(10): 0141842.

10. Dominguez-Bello MG, Costello EK, Contreras M, et al. Delivery mode shapes the acquisition and structure of the initial microbiota across multiple body habitats in newborns. Proceedings of the National Academy of Sciences. 2010;107(26):11971-5.

11. Sarkany I, Gaylarde CC. Bacterial colonisation of the skin of the newborn. The Journal of pathology and bacteriology. 1968;95(1):115-122.

12. Arzumanyan V, Magarshak O, Semenov B. Yeast fungi in patients with allergic disease: Species variety and sensitivity to antifungal drugs Bulletin of experimental biology and medicine. 2000;129(6):601-604.

13. Giacomoni PU, Mammone T, Teri M. Gender-linked differences in human skin. Journal of dermatological science. 2009;55(3):144-149.

14. Roth RR, James WD. Microbial ecology of the skin. Annual Reviews in Microbiology. 1988;42(1):441-464.

15. Hoy J, Hsu KC, Rolston K, et al. Iricnosporon beigelii Infection: A Review. Reviews of infectious diseases. 1986;8(6):959-967. 